LA-UR- $96-3447$

$$
\text { CONF- } 9609256--4
$$

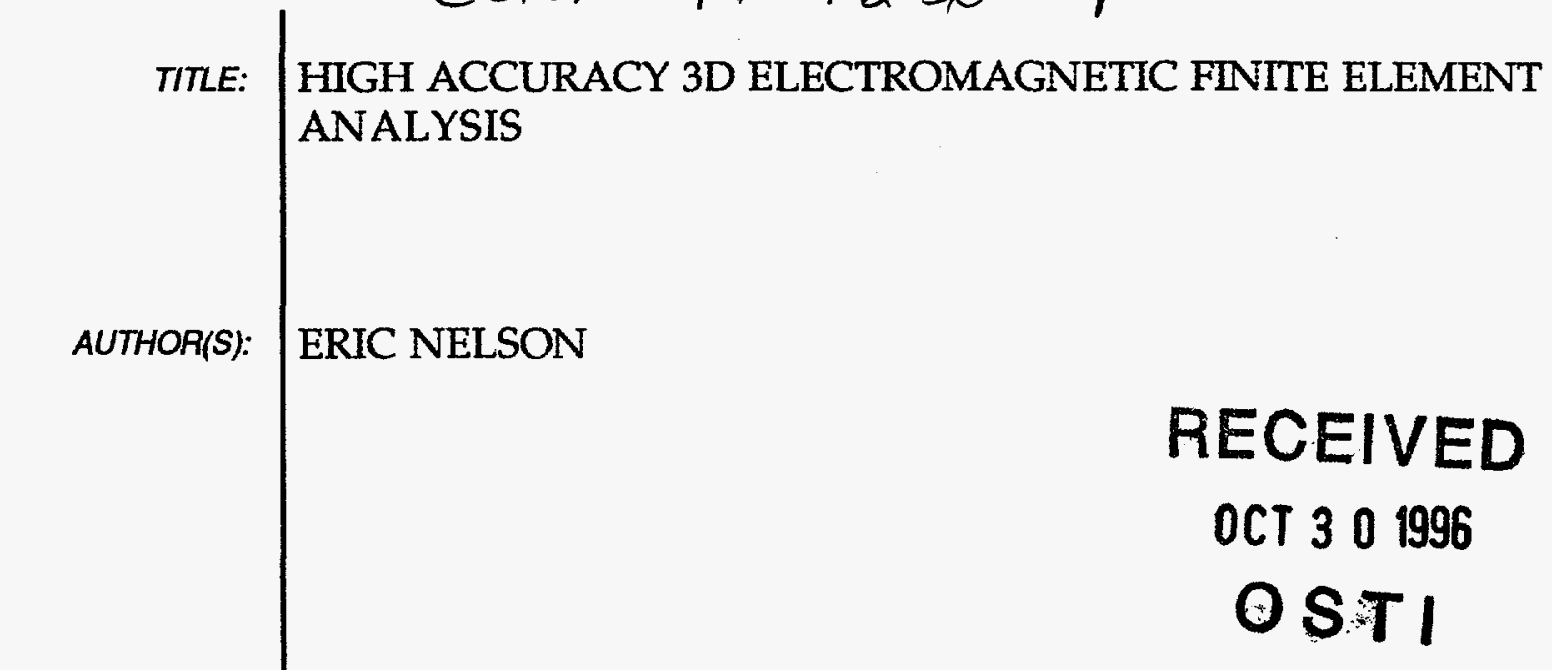

SUBMITTED TO:

COMPUTATIONAL ACCELERATOR PHYSICS 1996 SEPTEMBER 24-27, 1996

WILLIAMSBURG, VA

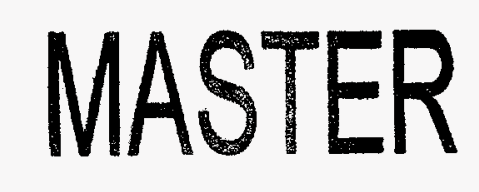

Los

Alamos

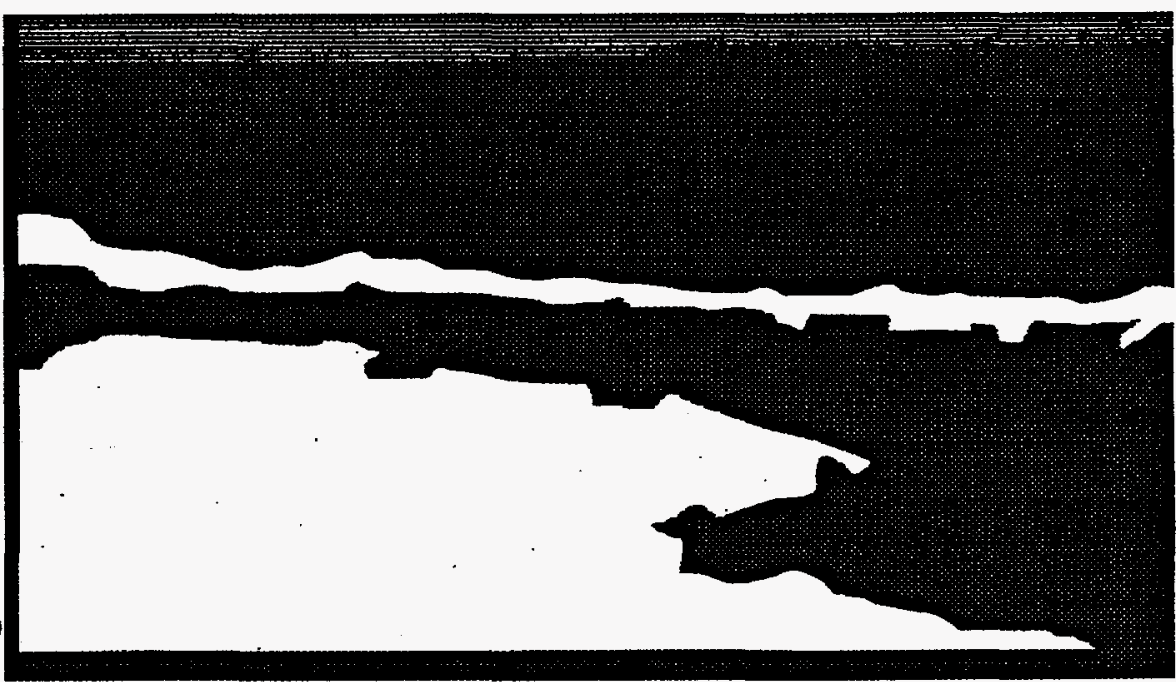

NATIONAL LABORATORY

Los Alamos National Laboratory, an affirmative action/equal opponunity employer, is operated by the University of California for the U.S. Department of Energy under contract W-7405-ENG-36. By acceptance of this article, the publisher recognizes that the U.S. Government retains a nonexclusive, royalty-free license to publish or reproduce the published form of this contribution, or to allow others to do so, for U.S. Government purposes. The Los Alamos National Laboratory requests that the publisher identify this article as work performed under the auspices of the U.S. Department of Energy. 


\section{DISCLAIMER}

This report was prepared as an account of work sponsored by an agency of the United States Government. Neither the United States Government nor any agency thereof, nor any of their employees, makes any warranty, express or implied, or assumes any legal liability or responsibility for the accuracy, completeness, or usefulness of any information, apparatus, product, or process disclosed, or represents that its use would not infringe privately owned rights. Reference herein to any specific commercial product, process, or service by trade name, trademark, manufacturer, or otherwise does not necessarily constitute or imply its endorsement, recommendation, or favoring by the United States Government or any agency thereof. The views and opinions of authors expressed herein do not necessarily state or reflect those of the United States Government or any agency thereof. 


\section{DISCLAIMER}

Portions of this document may be illegible in electronic image products. Images are produced from the best available original document. 


\title{
High Accuracy 3D Electromagnetic Finite Element Analysis
}

\author{
Eric M. Nelson \\ Los Alamos National Laboratory, \\ Los Alamos, New Mexico 87545
}

\begin{abstract}
A high accuracy 3D electromagnetic finite element field solver employing quadratic hexahedral elements and quadratic mixed-order one-form basis functions will be described. The solver is based on an object-oriented $\mathrm{C}++$ class library. Test cases demonstrate that frequency errors less than $10 \mathrm{ppm}$ can be achieved using modest workstations, and that the solutions have no contamination from spurious modes. The role of differential geometry and geometrical physics in finite element analysis will also be discussed.
\end{abstract}

\section{INTRODUCTION}

Electromagnetic finite element analysis (FEA) is becoming more popular as the accuracy and reliability of FEA codes improve. Accurate models of complicated structures have long been sought, and FEA with warped and/or unstructured meshes has been perceived as one path to achieve this accuracy. Thermal and mechanical FEA has met great success, but electromagnetic FEA has traditionally been plagued with reliability problems. Usually these reliability problems take the form of spurious modes (see (1) for many references). Fortunately, numerous workers have made steady progress in the past decade to eliminate these problems.

Differential geometry has been mentioned a few times $(1,2)$ in the electromagnetic FEA literature. It is an excellent tool for understanding electromagnetic FEA. Unfortunately, most of the current literature continues to use vector calculus notation, thus obscuring the simple nature of electromagnetic FEA.

Some reliable 3D electromagnetic FEA codes have existed for some time (see (3) and (4) for example). I have written an electromagnetic FEA code which should be equivalent to the code described in (4). In this paper I would like to (1) describe what I have learned about electromagnetic FEA from studying some differential geometry, and (2) demonstrate the accuracy and reliability of this FEA code.

* Work supported by DOE, contract W-7405-ENG-36. 


\section{FINITE ELEMENT FORMULATION}

In vector calculus notation, this FEA code is based on the following weak formulation of the eigenmode problem for electric fields: find eigenvalues $\omega^{2} / c^{2}$ and the corresponding eigenmode fields $\mathbf{E} \in \mathcal{U}_{E}$ such that $\forall \mathbf{F} \in \mathcal{U}_{E}$,

$$
\int_{\Omega}(\nabla \times \mathbf{F}) \cdot \mu^{-1}(\nabla \times \mathbf{E})-\frac{\omega^{2}}{c^{2}} \mathbf{F} \cdot \boldsymbol{\epsilon} \mathbf{E} d \Omega=0,
$$

where $\Omega$ is the cavity interior and the space $\mathcal{U}_{E}$ of test functions $\mathrm{F}$ and trial functions $\mathbf{E}$ is

$$
\mathcal{U}_{E}=\left\{\mathbf{E} \in \mathcal{H}_{\text {curl }}(\Omega): \hat{\mathbf{n}} \times \mathbf{E}=0 \text { on } d \Omega\right\},
$$

and $\mathcal{H}_{\text {curl }}(\Omega)$ is the space of vector fields on $\Omega$ which are square integrable in the following sense,

$$
\mathcal{H}_{\text {curl }}(\Omega)=\left\{\mathrm{E}: \int_{\Omega}|\nabla \times \mathbf{E}|^{2}+|\mathbf{E}|^{2} d \Omega \text { exists }\right\} .
$$

A similar formulation is based on the magnetic fields: find eigenvalues $\omega^{2} / c^{2}$ and the corresponding eigenmode fields $\mathbf{H} \in \mathcal{U}_{H}$ such that $\forall \mathbf{G} \in \mathcal{U}_{H}$,

$$
\int_{\Omega}(\nabla \times \mathbf{G}) \cdot \epsilon^{-1}(\nabla \times \mathbf{H})-\frac{\omega^{2}}{c^{2}} \mathbf{G} \cdot \boldsymbol{\mu} \mathbf{H} d \Omega=0,
$$

where the space $\mathcal{U}_{H}=\mathcal{H}_{\text {curl }}(\Omega)$.

The cavity interior $\Omega$ is partitioned into quadratic hexahedral (27-node) elements. Curved edges and faces allows these elements to closely follow curved boundaries. On each element there are 54 quadratic mixed-order 1-form basis functions, which are described in more detail below.

Numerical integration is used to compute the matrix components, and a simple subspace iteration scheme with a conjugate gradient solve is used to solve the sparse algebraic eigenvalue problem. A C++ class library handles matrices and bookkeeping of elements, faces, edges, nodes and basis functions.

\section{LESSONS FROM GEOMETRICAL PHYSICS}

In the finite element method, the problem domain $\Omega$ is divided into elements with simple shapes like tetrahedra and hexahedra. Each element $\Omega_{e}$ has a local coordinate system (i.e., a master element) and a map $x_{e}$ from local to global coordinates. This map is typically only used to define basis functions and to change variables to numerically integrate equation (1) over $\Omega_{e}$.

There is a close parallel between FEM and differential geometry. In differential geometry one considers a manifold (the problem domain $\Omega$ ) which is covered by coordinate patches (the elements). Where the coordinate patches 
overlap, the coordinate system of one patch is a differentiable function of the coordinates in the other patch. Differential geometry does not demand that a global coordinate system exist, but it accomodates one very well.

What does one learn from this comparison? First, the local coordinates of an element are a valid coordinate system. The physical equations can be expressed in local coordinates just like they are expressed in global coordinates.

Consider a vector basis function. There is no conceptual difference between a vector in global coordinates and a vector in local coordinates. The two are related by the transformation rule for vectors,

$$
v^{i}=\sum_{j=1}^{3} \frac{\partial x_{e}^{i}}{\partial u^{j}} \bar{v}^{j},
$$

where $v^{i}$ and $\bar{v}^{j}$ are the components of the vector in the global and local coordinate basis, respectively, and $x_{e}^{i}\left(u^{j}\right)$ is the map from local coordinates $u^{j}$ $(j=1,2,3)$ to global coordinates $x^{i}(i=1,2,3)$. Note that early attempts at $3 \mathrm{D}$ electromagnetic FEA violated this transformation rule. They would use scalar basis functions for the vector components, and simply map these components from local to global coordinates, $v^{i}=\bar{v}^{i}$. This procedure is geometrically incorrect, and thus the results of these FEA codes were usually flawed.

Now consider which basis functions are appropriate for electromagnetic FEA. In reference (5), Maxwell's equations are described in terms of differential geometry and geometrical physics. In particular, it is pointed out that the electric field is most naturally expressed as a 1-form, or covariant vector. Hence the appropriate basis functions for electric fields is most easily expressed as 1forms, not vectors. Recent literature typically shows complicated constructions for these basis functions (see references (1) and (6) for example), but if one writes the basis functions as 1-forms in local coordinates $(u, v$ and $w)$, they are simple polynomials. This is the spirit of FEM - the field is a linear combination of simple basis functions on simple elements.

The choice of basis functions is important, as described in (7) and (8). The basis functions should be mixed-order, with the basis for the field along a coordinate direction, say $E_{u}$, being complete to order $p$ in $v$ and $w$, but only $p-1$ in $u$. The basis functions employed in this FEA are listed in table 1 . The local coordinates of the hexahedra are $0 \leq u \leq 1,0 \leq v \leq 1$ and $0 \leq w \leq 1$, and the coordinate basis for 1-forms is $d u, d v$ and $d w$.

The basis functions are assembled so that the tangential component of the field at an interface between elements is continuous. Thinking of the basis functions as 1-forms makes it easy to verify that the assembly process works. The basis functions are characterized by their non-zero tangential field on an edge or face. The first 12 basis functions have constant tangential field along one edge. These basis functions, by themselves, are appropriate for a linear mixed-order approximation to the fields. The next 12 basis functions $\left(a_{13}\right.$ 
Table 1. Quadratic 1-form basis functions $a_{i}$ for hexahedral elements.

$$
\begin{aligned}
& a_{1}=(1-v)(1-w) d u \quad a_{5}=(1-u)(1-v) d w \quad a_{9}=(1-v) w d u \\
& a_{2}=(1-u)(1-w) d v \quad a_{6}=u(1-v) d w \quad a_{10}=(1-u) w d v \\
& a_{3}=u(1-w) d v \quad a_{7}=(1-u) v d w \quad a_{11}=u w d v \\
& a_{4}=v(1-w) d u \quad a_{8}=u v d w \quad a_{12}=v w d u \\
& a_{13}=(2 u-1) a_{1} \quad a_{17}=(2 w-1) a_{5} \quad a_{21}=(2 u-1) a_{9} \\
& a_{14}=(2 v-1) a_{2} \quad a_{18}=(2 w-1) a_{6} \quad a_{22}=(2 v-1) a_{10} \\
& a_{15}=(2 v-1) a_{3} \quad a_{19}=(2 w-1) a_{7} \quad a_{23}=(2 v-1) a_{11} \\
& a_{16}=(2 u-1) a_{4} \quad a_{20}=(2 w-1) a_{8} \quad a_{24}=(2 u-1) a_{12} \\
& a_{25}=4 u(1-u)(1-w) d v \quad a_{31}=u 4 w(1-w) d v \\
& a_{26}=4 v(1-v)(1-w) d u \quad a_{32}=u 4 v(1-v) d w \\
& a_{27}=(1-v) 4 w(1-w) d u \quad a_{33}=4 u(1-u) v d w \\
& a_{28}=4 u(1-u)(1-v) d w \quad a_{34}=v 4 w(1-w) d u \\
& a_{29}=(1-u) 4 v(1-v) d w \quad a_{35}=4 v(1-v) w d u \\
& a_{30}=(1-u) 4 w(1-w) d v \quad a_{36}=4 u(1-u) w d v \\
& a_{37}=(2 v-1) a_{25} \quad a_{41}=(2 w-1) a_{29} \quad a_{45}=(2 w-1) a_{33} \\
& a_{38}=(2 u-1) a_{26} \quad a_{42}=(2 v-1) a_{30} \quad a_{46}=(2 u-1) a_{34} \\
& a_{39}=(2 u-1) a_{27} \quad a_{43}=(2 v-1) a_{31} \quad a_{47}=(2 u-1) a_{35} \\
& a_{40}=(2 w-1) a_{28} \quad a_{44}=(2 w-1) a_{32} \quad a_{48}=(2 v-1) a_{36} \\
& a_{49}=4 v(1-v) 4 w(1-w) d u \quad a_{52}=(2 u-1) a_{49} \\
& a_{50}=4 u(1-u) 4 w(1-w) d v \quad a_{53}=(2 v-1) a_{50} \\
& a_{51}=4 u(1-u) 4 v(1-v) d w \quad a_{54}=(2 w-1) a_{51}
\end{aligned}
$$

to $a_{24}$ ) have linear tangential field along one edge. The next 24 basis functions $\left(a_{25}\right.$ to $a_{48}$ ) have non-zero tangential field on one face but no edges. Finally, the last 6 basis functions ( $a_{49}$ to $a_{54}$ ) have no tangential field on the element boundary.

To assemble global basis functions, the first 24 basis functions must coordinate with all elements which share their one edge. The second 24 basis functions must coordinate with the element which shares their one face. The last 6 basis functions are valid global basis functions by themselves, and need not coordinate with any neighboring elements.

In terms of exterior products $(\wedge)$ and exterior derivatives $(d)$, equation $(1)$ can be expressed as

$$
\int_{\Omega} d F \wedge \mu^{-1} d E-\frac{\omega^{2}}{c^{2}} \mathbf{F} \wedge \epsilon E=0
$$

where $\boldsymbol{\mu}$ and $\epsilon$ are now Hodge-star operators which convert 1-forms (e.g., $E$ and $H$ ) to 2-forms (e.g., $D$ and $B$ ) using some tensor (i.e., the permittivity and 


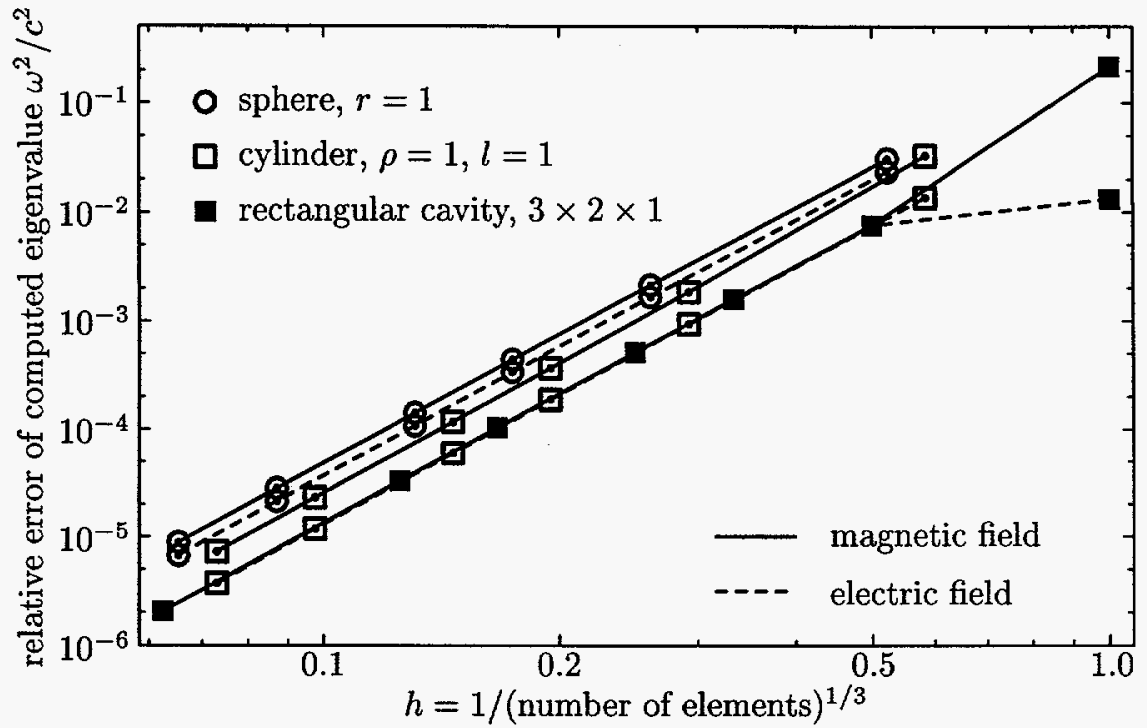

Figure 1. Relative eigenvalue error versus element size $h$ for three test cavities.

permeability tensors). Differential geometry reminds us that we can differentiate in local coordinates (where the basis and their derivatives are simple) and transform the result to global coordinates (where the permittivity, permeability and metric tensors are usually simple). This avoids messy applications of the chain rule and extra calculation. In some cases it may be cost-effective to transform the tensors to local coordinates and perform all of the computations in local coordinates.

\section{TESTS OF THE FEA CODE}

This FEA code was tested on (1) a $3 \times 2 \times 1$ rectangular cavity, (2) a pillbox cavity with radius $\rho=1$ and height $l=1$ and (3) a $\rho=1$ spherical cavity. The mesh was refined in a regular manner from a coarse mesh to a fine mesh. The relative error of the computed eigenvalues for both electric and magnetic field calculations is shown in figure 1. Excellent accuracies (less than 10ppm error) are achieved on a modest workstation. The error is proportional to $h^{4}$, where $h$ is the element size. The numerical eigenvalue of the spurious modes is less than $10^{-12}$, so spurious modes are well separated from the physical modes.

Test cases with inhomogeneously filled cavities show similar results. The spurious modes still have zero eigenvalue, even when the dielectric properties change within an element. The accuracy is excellent, with the caveat that sharp corners cause a significant reduction in accuracy, so the mesh needs to be refined in these locations.

The execution times are reasonable when compared with MAFIA. Figure 2 compares this FEA code (YAP) with MAFIA on a pillbox test cavity. A uniform grid was employed in the MAFIA calculations in order to produce the typical accuracy achieved by MAFIA in a more complicated structure. This 


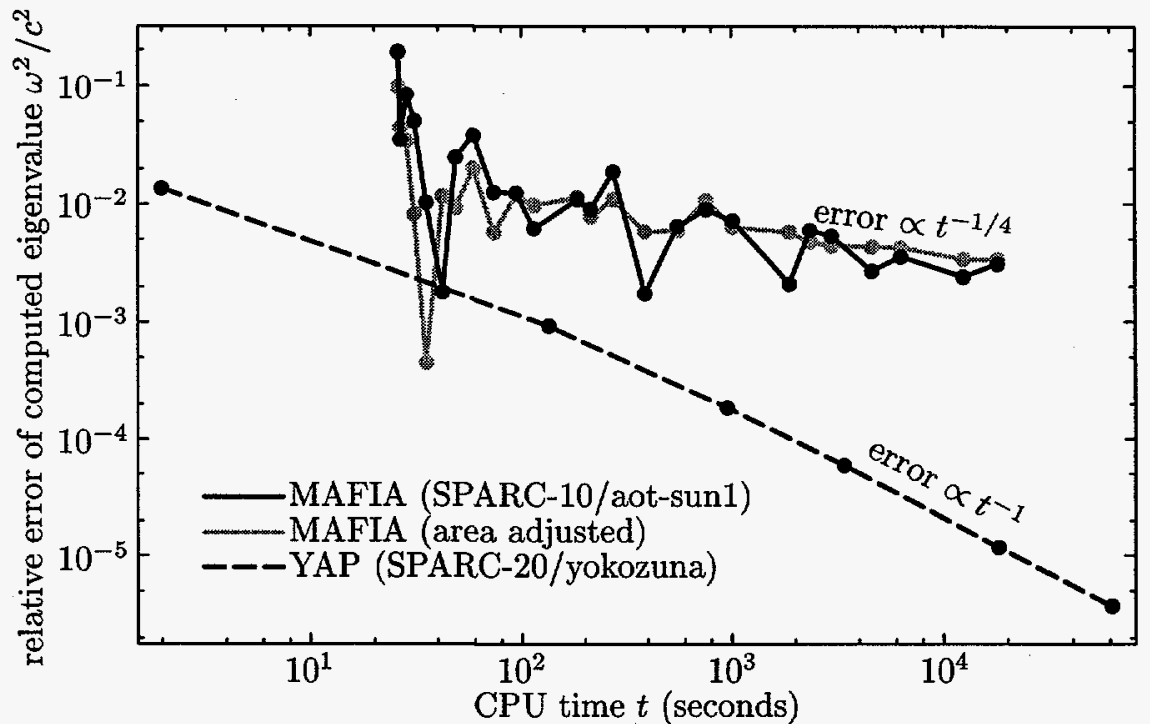

Figure 2. Relative eigenvalue error versus CPU time for MAFIA and YAP. The test structure is a pillbox cavity with radius $\rho=1$ and height $l=1$. The YAP results are electric field calculations. The MAFIA results employed a uniform grid.

FEA excels when good accuracy is desired since the FEA error scales like $t^{-1}$ instead of $t^{-1 / 4}$. This difference is due to the discretization error (proportional to $h^{4}$ for this FEA, and $h$ for MAFIA).

\section{CONCLUSION}

A 3D electromagnetic finite element analyis code with high accuracy and with no contamination from spurious modes has been demonstrated. Differential geometry and geometrical physics is useful for understanding the features of these codes and why they work.

\section{References}

1. Cendes, Z. J., IEEE Trans. Magnetics 27, 3958-3966 (1991).

2. Bossavit, A., IEE Proc. 135, 493-500 (1988).

3. Barton, M. L. and Cendes, Z. J., J. Appl. Phys. 61, 3919-3921 (1987).

4. Crowley, C. W. et. al., IEEE Trans. Magnetics 24, 397-400 (1988).

5. Deschamps, G. A., Proc. IEEE 69, 676-696 (1981).

6. Nelson, E. M., Ph.D. thesis, Stanford University (1993); also SLAC-431.

7. Nédélec, J. C., Numer. Math. 35, 315-341 (1980).

8. Nédélec, J. C., Numer. Math. 50, 57-81 (1986). 